\title{
CONFIGURING A SENSOR NETWORK FOR FAULT DETECTION IN DISTRIBUTED PARAMETER SYSTEMS
}

\author{
MACIEJ PATAN, DARIUSZ UCIŃSKI \\ Institute of Control and Computation Engineering \\ University of Zielona Góra, ul. Podgórna 50, 65-246 Zielona Góra, Poland \\ e-mail: \{M.Patan, D.Ucinski\}@issi.uz.zgora.pl
}

\begin{abstract}
The problem of fault detection in distributed parameter systems (DPSs) is formulated as that of maximizing the power of a parametric hypothesis test which checks whether or not system parameters have nominal values. A computational scheme is provided for the design of a network of observation locations in a spatial domain that are supposed to be used while detecting changes in the underlying parameters of a distributed parameter system. The setting considered relates to a situation where from among a finite set of potential sensor locations only a subset can be selected because of the cost constraints. As a suitable performance measure, the $D_{s}$-optimality criterion defined on the Fisher information matrix for the estimated parameters is applied. Then, the solution of a resulting combinatorial problem is determined based on the branch-and-bound method. As its essential part, a relaxed problem is discussed in which the sensor locations are given $a$ priori and the aim is to determine the associated weights, which quantify the contributions of individual gauged sites. The concavity and differentiability properties of the criterion are established and a gradient projection algorithm is proposed to perform the search for the optimal solution. The delineated approach is illustrated by a numerical example on a sensor network design for a two-dimensional convective diffusion process.
\end{abstract}

Keywords: branch-and-bound, constrained experimental design, distributed parameter systems, fault detection, parameter estimation, sensor location.

\section{Introduction}

Experimental design for spatio-temporal physical systems (especially, in environmental protection, nuclear power engineering, oil industry, etc.) is often related to an optimal choice of measurement conditions in order to obtain the best information for estimating unknown parameters which can then be used in optimal control. The impossibility to observe the system states over the entire spatial domain implies the question of where to locate discrete sensors so as to accurately estimate the unknown system parameters. An example which is particularly stimulating in the light of the results reported in this paper is the optimization of air quality monitoring networks. The question of how to optimize sensor locations acquires especially vital importance in the context of recent advances in distributed sensor networks (Cassandras and Li, 2005).

Over the past years, laborious research on the development of strategies for efficient sensor placement has been conducted (for reviews, see the papers (Kubrusly and Malebranche, 1985; Uciński, 1992; Uciński, 2000; van de
Wal and de Jager, 2001) and the comprehensive monographs (Uciński, 2005; Uciński, 1999)). Nevertheless, although the need for systematic methods was widely recognized, most techniques communicated by various authors usually rely on an exhaustive search over a predefined set of candidates and the combinatorial nature of the design problem is taken into account very occasionally (van de Wal and de Jager, 2001). Needless to say, this approach, which is feasible for a relatively small number of possible locations, soon becomes useless as the number of possible location candidates increases.

Additionally, in spite of the rapid development of fault detection and localization methods for dynamic systems (Korbicz et al., 2004; Isermann, 1997; Patton and Korbicz, 1999; Patton et al., 2000; Chiang et al., 2001), there are no effective methods tailored to spatiotemporal systems. Some successful attempts at exploiting the $D_{s}$-optimality criterion were reported by Patan and Patan (2005). The aim of the research reported there was to develop a practical approach to sensor selection for fault detection which, while being independent of a particular 
model of the dynamic DPS in question, would be versatile enough to cope with practical monitoring networks consisting of many stationary sensors. Specifically, we consider $N$ possible sites at which to locate a sensor, but limitations on the number of sensors at our disposal allow only $n$ of them (typically, $n$ is much smaller than $N$ ) to be selected. Consequently, the problem is to divide the $N$ available sites between $n$ gauged sites and the remaining $N-n$ ungauged sites so as to maximize the determinant of the Fisher information matrix (FIM) associated with the parameters to be estimated. Since selecting the best subset of sites to locate the sensors constitutes an inherently discrete large-scale resource allocation problem whose solution may be prohibitively time-consuming, an efficient guided search algorithm based on the branch-and-bound method is developed, which implicitly enumerates all the feasible sensor configurations, using relaxed optimization problems that involve no integer constraints.

The idea of using branch-and-bound (BB) for sensor selection in a DPS was already applied by Uciński and Patan (2007) in the context of $D$-optimality. Obviously, BB constitutes one of the most frequent approaches to solve discrete optimization problems and it has indeed been used in the context of network design (Boer et al., 2001). Nevertheless, the main contribution of this paper consists in creative usage of a simple and powerful computational scheme to obtain upper bounds to the optimal values of the $D_{s}$-optimality criterion, which proves especially attractive in the light of applications in fault detection and diagnosis. Moreover, various properties of the $D_{s}$-optimality criterion are given and a relaxed problem is introduced whose solution is obtained through a gradient projection algorithm.

The paper has the following structure: Section 2 states formally the sensor network design problem as a discrete resource allocation problem. The general BB scheme for its solution is discussed in Sec. 3. Section 4 describes the algorithmic solution for computing upper bounds required by the branching rule. In Sec. 5, we illustrate the use of our algorithm using the example of a sensor network design problem regarding a two-dimensional convective diffusion process. We conclude in Sec. 6 with some comments on related open problems. The proofs of some essential properties of the $D_{s}$-optimality criterion are contained in Appendix.

Notation. Throughout the paper, $\mathbb{R}_{+}$and $\mathbb{R}_{++}$stand for the sets of nonnegative and positive real numbers, respectively. We adopt the convention that all vectors have the column form. The set of real $m \times n$ matrices is denoted by $\mathbb{R}^{m \times n}$. We use $\mathbb{S}^{m}$ to denote the set of symmetric $m \times m$ matrices, $\mathbb{S}_{+}^{m}$ to denote the set of symmetric nonnegative definite $m \times m$ matrices, and $\mathbb{S}_{++}^{m}$ to denote the set of symmetric positive definite $m \times m$ matrices. The curled inequality symbol $\succeq$ (resp. $\succ$ ) is used to denote gener- alized inequalities. More precisely, between vectors, it represents a componentwise inequality, and between symmetric matrices, it represents the Löwner ordering: given $A, B \in \mathbb{S}^{m}, A \succeq B$ means that $A-B$ is nonnegative definite (resp. positive definite).

\section{Sensor selection for fault detection}

Consider a bounded spatial domain $\Omega \subset \mathbb{R}^{d}$ with sufficiently smooth boundary $\Gamma$, a bounded time interval $T=\left(0, t_{f}\right]$, and a distributed parameter system whose scalar state at a spatial point $x \in \bar{\Omega} \subset \mathbb{R}^{d}$ and time instant $t \in \bar{T}$ is denoted by $y(x, t)$. Mathematically, the system state is governed by the partial differential equation (PDE)

$$
\frac{\partial y}{\partial t}=\mathcal{F}(x, t, y, \theta) \quad \text { in } \Omega \times T,
$$

where $\mathcal{F}$ is a well-posed, possibly nonlinear, differential operator which involves first- and second-order spatial derivatives and may include terms accounting for forcing inputs specified a priori. The PDE (1) is accompanied by the appropriate boundary and initial conditions,

$$
\begin{aligned}
\mathcal{B}(x, t, y, \theta) & =0 & & \text { on } \Gamma \times T, \\
y & =y_{0} & & \text { in } \Omega \times\{t=0\},
\end{aligned}
$$

respectively, $\mathcal{B}$ being an operator acting on the boundary $\Gamma$ and $y_{0}=y_{0}(x)$ a given function. Conditions (2) and (3) complement (1) such that the existence of a sufficiently smooth and unique solution is guaranteed. We assume that the forms of $\mathcal{L}$ and $\mathcal{B}$ are given explicitly up to an $m$-dimensional vector of unknown constant parameters $\theta$ which must be estimated using observations of the system. The implicit dependence of the state $y$ on the parameter vector $\theta$ will be be reflected by the notation $y(x, t ; \theta)$.

In what follows, we consider the discrete-continuous observations provided by $n$ stationary pointwise sensors, namely,

$$
z_{\mathrm{m}}^{\ell}(t)=y\left(x^{\ell}, t ; \theta\right)+\varepsilon\left(x^{\ell}, t\right), \quad t \in T,
$$

where $z_{\mathrm{m}}^{\ell}(t)$ is the scalar output and $x^{\ell} \in X$ stands for the location of the $\ell$-th sensor $(\ell=1, \ldots, n), X$ signifies the part of the spatial domain $\Omega$ where the measurements can be made and $\varepsilon\left(x^{\ell}, t\right)$ denotes the measurement noise. This relatively simple conceptual framework involves no loss of generality since it can be easily generalized to incorporate, e.g., multiresponse systems or inaccessibility of state measurements, cf. (Uciński, 2005, p. 95).

It is customary to assume that the measurement noise is zero-mean, Gaussian, spatial uncorrelated and white (Quereshi et al., 1980; Omatu and Seinfeld, 1989; Amouroux and Babary, 1988), i.e.,

$$
\mathrm{E}\left\{\varepsilon\left(x^{\ell}, t\right) \varepsilon\left(x^{\ell^{\prime}}, t^{\prime}\right)\right\}=\sigma^{2} \delta_{\ell \ell^{\prime}} \delta\left(t-t^{\prime}\right),
$$


where $\sigma^{2}$ defines the intensity of the noise, $\delta_{i j}$ and $\delta(\cdot)$ standing for the Kronecker and Dirac delta functions, respectively. Although white noise is a physically impossible process, it constitutes a reasonable approximation to a disturbance whose adjacent samples are uncorrelated at all time instants for which the time increment exceeds some value that is small compared with the time constants of the DPS. A rigorous formulation for a time-correlated setting (cf. Appendix C1 of (Uciński, 2005)) is well beyond the mathematical framework of this paper, but the attendant difficulties are mainly technical and do not substantially affect the basic results to be obtained. What is more, the white-noise assumption is consistent with most of the literature on the subject.

The most widely used formulation of the parameter estimation problem is as follows: Given the model (1)(3) and the outcomes of the measurements $z_{\mathrm{m}}^{\ell}(\cdot), \ell=$ $1, \ldots, n$, estimate $\theta$ by $\widehat{\theta}$, a global minimizer of the output least-squares error criterion

$$
\mathcal{J}(\vartheta)=\sum_{\ell=1}^{n} \int_{T}\left\{z_{\mathrm{m}}^{\ell}(t)-y\left(x^{\ell}, t ; \vartheta\right)\right\}^{2} \mathrm{~d} t,
$$

where $y(\cdot, \cdot ; \vartheta)$ denotes the solution to (1)-3) for a given value of the parameter vector $\vartheta$.

The basic idea of fault detection is to compare the resulting parameter estimates with the corresponding known nominal values, treating possible differences as residuals which contain information about potential faults. Based on some thresholding techniques, the appropriate decision making system could be constructed to detect abnormal situations in system functioning (Patan and Patan, 2005).

Basically, only some parameters can be useful for the diagnosis. This accounts for partitioning the parameter vector into two two subsets. With no loss of generality, we may write

$$
\theta=\left[\begin{array}{lll:lll}
\theta_{1} & \ldots & \theta_{s} & \theta_{s+1} & \ldots & \theta_{m}
\end{array}\right]^{\top}=\left[\begin{array}{l:l}
\alpha^{\top} & \beta^{\top}
\end{array}\right],
$$

where $\alpha$ is a vector of $s$ parameters which are essential for a proper fault detection and $\beta$ is the vector of some unknown parameters which are a part of the model but are useless for fault detection. Based on the observations, it is possible to test the simple null hypothesis

$$
H_{0}: \alpha=\alpha^{0},
$$

where $\alpha^{0}$ is the nominal value for the vector $\alpha$ corresponding to the normal system performance.

For a fixed significance level (i.e., fixed probability of rejecting $H_{0}$ when it is true), the power of the likelihood ratio test for the alternative hypothesis $H_{A}: \alpha \neq \alpha^{*}$ (i.e., 1 - the probability of accepting $H_{0}$ when $H_{A}$ is true) can be made large by maximizing the $D_{s}$-optimality criterion (see (Patan and Patan, 2005) for details):

$$
\Psi_{s}[M]=\log \operatorname{det}\left[M_{\alpha \alpha}-M_{\alpha \beta} M_{\beta \beta}^{-1} M_{\alpha \beta}^{\top}\right],
$$

where $M \in \mathbb{R}^{m \times m}$ stands for the so-called Fisher information matrix, which is decomposed as

$$
M=\left[\begin{array}{c:c}
M_{\alpha \alpha} & M_{\alpha \beta} \\
\hdashline M_{\alpha \beta}^{\mathrm{T}} & M_{\beta \beta}
\end{array}\right]
$$

such that $M_{\alpha \alpha} \in \mathbb{R}^{s \times s}, M_{\alpha \beta} \in \mathbb{R}^{s \times(m-s)}, M_{\beta \beta} \in$ $\mathbb{R}^{(m-s) \times(m-s)}$. The FIM is widely used in optimum experimental design theory for lumped systems (Fedorov and Hackl, 1997; Pázman, 1986; Pukelsheim, 1993; Walter and Pronzato, 1997; Atkinson et al., 2007). In our setting, the FIM is given by (Quereshi et al., 1980)

$$
M\left(x^{1}, \ldots, x^{n}\right)=\sum_{\ell=1}^{n} \int_{T} g\left(x^{\ell}, t\right) g^{\top}\left(x^{\ell}, t\right) \mathrm{d} t,
$$

where

$$
g(x, t)=\left[\begin{array}{lll}
\frac{\partial y(x, t ; \vartheta)}{\partial \vartheta_{1}}, & \ldots, & \frac{\partial y(x, t ; \vartheta)}{\partial \vartheta_{m}}
\end{array}\right]_{\vartheta=\theta^{0}}^{\top}
$$

stands for the so-called sensitivity vector, $\theta^{0}$ being the nominal value of the parameter vector $\theta$ (Uciński, 2005; Sun, 1994; Rafajłowicz, 1981; Rafajłowicz, 1983). Up to a constant scalar multiplier, the inverse of the FIM constitutes a good approximation of $\operatorname{cov}(\widehat{\theta})$ provided that the time horizon is large, the nonlinearity of the model with respect to its parameters is mild, and the measurement errors are independently distributed and have small magnitudes (Walter and Pronzato, 1997; Fedorov and Hackl, 1997).

Observe that for the partition

$$
M^{-1}=\left[\begin{array}{c:c}
D_{\alpha \alpha} & D_{\alpha \beta} \\
\hdashline D_{\alpha \beta}^{\top} & D_{\beta \beta}
\end{array}\right],
$$

where $D_{\alpha \alpha} \in \mathbb{R}^{s \times s}, D_{\alpha \beta} \in \mathbb{R}^{s \times(m-s)}, D_{\beta \beta} \in$ $\mathbb{R}^{(m-s) \times(m-s)}$, we have (Bernstein, 2005, Fact 2.8.7, p.44)

$$
D_{\alpha \alpha}=\left(M_{\alpha \alpha}-M_{\alpha \beta} M_{\beta \beta}^{-1} M_{\alpha \beta}^{\top}\right)^{-1}
$$

and further (Bernstein, 2005, Fact 2.15.8, p.73)

$$
\operatorname{det}\left(D_{\alpha \alpha}\right)=\frac{\operatorname{det}\left(M_{\beta \beta}\right)}{\operatorname{det}(M)} .
$$

Consequently, the maximization of the $D_{s^{-}}$ optimality criterion amounts to the minimization of $\operatorname{det}\left(D_{\alpha \alpha}\right)$, which is proportional to the determinant of the covariance matrix for $\alpha$.

The optimal sensor location problem considered in what follows is: Given a set of $N$ possible candidate sensor locations, where $N>n$, we wish to seek the best subset of $n$ locations from among the $N$ possible, so that the problem is then reduced to a combinatorial one. In other 
words, the problem is to divide the $N$ available sites between $n$ gauged sites and the remaining $N-n$ ungauged sites so as to maximize the determinant of the FIM associated with the parameters to be estimated. This formulation will be also adopted here.

Specifically, let $x^{i}, i=1, \ldots, N$ denote the positions of sites where sensors can potentially be placed. Now that our design criterion has been established, the problem is to find an optimal allocation of $n$ available sensors to $x^{i}$, $i=1, \ldots, N$ so as to maximize the value of the design criterion incurred by the allocation. In order to formulate this mathematically, for each possible location $x^{i}$ introduce a variable $v_{i}$ which takes the value 1 or 0 depending on whether a sensor is or is not located at $x^{i}$, respectively. The FIM in (11) can then be rewritten as

$$
M\left(v_{1}, \ldots, v_{N}\right)=\sum_{i=1}^{N} v_{i} M_{i},
$$

where

$$
M_{i}=\int_{T} g\left(x^{i}, t\right) g^{\top}\left(x^{i}, t\right) \mathrm{d} t .
$$

It is straightforward to verify that the $m \times m$ matrices $M_{i}$ are nonnegative definite and, therefore, so is $M\left(v_{1}, \ldots, v_{N}\right)$.

Then our design problem takes the following form:

Problem P. Find a sequence $v=\left(v_{1}, \ldots, v_{N}\right)$ to maximize

$$
\mathcal{P}(v)=\Psi(M(v))
$$

subject to the constraints

$$
\begin{aligned}
\sum_{i=1}^{N} v_{i} & =n, \\
v_{i} & =0 \text { or } 1, \quad i=1, \ldots, N .
\end{aligned}
$$

This constitutes a $0-1$ integer programming problem which necessitates an ingenious solution. In (Uciński and Patan, 2007), a general computational scheme is proposed to solve this problem based on the branch-andbound method, which is a standard technique for such a class of tasks. Its presentation constitutes the next section of the paper.

\section{Branch-and-bound solution}

3.1. Outline. Branch-and-bound constitutes a general algorithmic technique for finding optimal solutions of various optimization problems, especially discrete or combinatorial (Floudas, 2001; Bertsekas, 1999). If applied carefully, it can lead to algorithms that run reasonably fast on average.

Principally, the BB method is a tree-search algorithm combined with a rule for pruning subtrees. Suppose we wish to maximize an objective function $\mathcal{P}(v)$ over a finite set $V$ of admissible values of the argument $v$ called the feasible region. BB then progresses by iteratively applying two procedures: branching and bounding. Branching starts with smartly covering the feasible region by two or more smaller feasible subregions (ideally, partitioning into disjoint subregions). It is then repeated recursively to each of the subregions until no more division is possible, which leads to a progressively finer partition of $V$. The consecutively produced subregions naturally generate a tree structure called the BB tree. Its nodes correspond to the constructed subregions, with the feasible set $V$ as the root node and the singleton solutions $\{v\}, v \in V$ as terminal nodes. In turn, the core of bounding is a fast method of finding upper and lower bounds to the maximum value of the objective function over a feasible subdomain. The idea is to use these bounds to economize computation by eliminating nodes of the $\mathrm{BB}$ tree that have no chance of containing an optimal solution. If the upper bound for a subregion $V_{A}$ from the search tree is lower than the lower bound for any other (previously examined) subregion $V_{B}$, then $V_{A}$ and all its descendant nodes may be safely discarded from the search. This step, termed pruning, is usually implemented by maintaining a global variable that records the maximum lower bound encountered among all subregions examined so far. Any node whose upper bound is lower than this value need not be considered further and thereby can be eliminated. It may happen that the lower bound for a node matches its upper bound. That value is then the maximum of the function within the corresponding subregion and the node is said to be solved. The search proceeds until all nodes have been solved or pruned, or until some specified threshold is met between the best solution found and the upper bounds on all unsolved problems.

3.2. Relaxed sensor selection problem. Let $I$ denote the index set $\{1, \ldots, N\}$ of possible sensor locations. Consider a slight modification of Problem P, which starts by replacing the feasible set

$$
V=\left\{\left(v_{1}, \ldots, v_{N}\right) \mid \sum_{i=1}^{N} v_{i}=n, v_{i}=0 \text { or } 1, \forall i \in I\right\}
$$

with

$$
\begin{aligned}
& V\left(I_{0}, I_{1}\right) \\
& =\left\{v \in V \mid v_{i}=0, \forall i \in I_{0}, v_{i}=1, \forall i \in I_{1}\right\},
\end{aligned}
$$

where $I_{0}$ and $I_{1}$ are disjoint subsets of $I$. Consequently, $V\left(I_{0}, I_{1}\right)$ is the subset of $V$ such that a sensor is placed at the locations with indices in $I_{1}$, no sensor is placed at the locations with indices in $I_{0}$, and a sensor may or may not be placed at the remaining locations. 
Then we introduce the following relaxed problem:

Problem $\mathbf{R}\left(\boldsymbol{I}_{0}, \boldsymbol{I}_{1}\right)$. Find a sequence $\bar{v}$ to maximize (18) subject to the constraints

$$
\begin{aligned}
& \sum_{i=1}^{N} v_{i}=n, \\
& v_{i}=0, \quad i \in I_{0}, \\
& v_{i}=1, \quad i \in I_{1}, \\
& 0 \leq v_{i} \leq 1, \quad i \in I \backslash\left(I_{0} \cup I_{1}\right) .
\end{aligned}
$$

In Problem $\mathrm{R}\left(I_{0}, I_{1}\right)$, all 0-1 constraints on the variables $v_{i}$ are relaxed by allowing them to take any value in the interval $[0,1]$, except that the variables $v_{i}, i \in I_{0} \cup I_{1}$ are fixed at either 0 or 1 .

Each subset $V\left(I_{0}, I_{1}\right)$ can be identified with a node in the BB tree. The key assumption in the BB method is that for every nonterminal node $V\left(I_{0}, I_{1}\right)$, i.e., the node for which $I_{0} \cup I_{1} \neq I$, there is an algorithm that determines an upper bound $\overline{\mathcal{P}}\left(I_{0}, I_{1}\right)$ to the maximum design criterion over $V\left(I_{0}, I_{1}\right)$, i.e.,

$$
\overline{\mathcal{P}}\left(I_{0}, I_{1}\right) \geq \max _{v \in V\left(I_{0}, I_{1}\right)} \mathcal{P}(v),
$$

and a feasible solution $\underline{v} \in V$ for which $\mathcal{P}(\underline{v})$ can serve as a lower bound to the maximum design criterion over $V$. Furthermore, we may compute $\overline{\mathcal{P}}\left(I_{0}, I_{1}\right)$ by solving relaxed Problem $\mathrm{R}\left(I_{0}, I_{1}\right)$. A simple and efficient method for its solution based on the gradient projection technique constitutes Section 4 of the paper. As a result of its application, we set $\overline{\mathcal{P}}\left(I_{0}, I_{1}\right)=\mathcal{P}(\bar{v})$.

As for $\underline{v}$, we can specify it as the best feasible solution (i.e., an element of $V$ ) found so far. If no solution has been found yet, we can either set the lower bound to $-\infty$, or use an initial guess about the optimal solution (experience provides evidence that the latter choice leads to a much more rapid convergence).

3.3. Branching rule. The result of solving Problem $\mathrm{R}\left(I_{0}, I_{1}\right)$ can serve as a basis to construct a branching rule for the binary $\mathrm{BB}$ tree. We adopt here the approach in which the node/subset $V\left(I_{0}, I_{1}\right)$ is expanded (i.e., partitioned) by first picking out all fractional values from among the values of the relaxed variables, and then rounding to 0 and 1 a value which is the most distant from both 0 and 1 . Specifically, we apply the following steps:

(i) Determine

$$
i_{\star}=\arg \min _{i \in I \backslash\left(I_{0} \cup I_{1}\right)}\left|v_{i}-0.5\right| .
$$

(In case there are several minimizers, randomly pick one of them.) (ii) Partition $V\left(I_{0}, I_{1}\right)$ into $V\left(I_{0} \cup\left\{i_{\star}\right\}, I_{1}\right)$ and $V\left(I_{0}, I_{1} \cup\left\{i_{\star}\right\}\right)$ whereby two descendants of the node in question are defined.

A recursive application of the branching rule starts from the root of the $\mathrm{BB}$ tree, which corresponds to the trivial subset $V(\emptyset, \emptyset)=V$ and the fully relaxed problem. Each node of the BB tree corresponds to a continuous relaxed problem, $\mathrm{R}\left(I_{0}, I_{1}\right)$, while each edge corresponds to fixing one relaxed variable at 0 or 1 .

The above scheme has to be complemented with a search strategy to incrementally explore all the nodes of the BB tree. Here we use a common depth-first technique (Reinefeld, 2001; Russell and Norvig, 2003), which always expands the deepest node in the current fringe of the search tree. The reason behind this decision is that the search proceeds immediately to the deepest level of the search tree, where the nodes have no successors (Gerdts, 2005). In this way, lower bounds on the optimal solution can be found or improved as fast as possible.

A recursive version of the resulting depth-first branch-and-bound is implemented in Algorithm 1 The operators involved in this implementation are as follows:

- Relaxed-Solution $\left(I_{0}, I_{1}\right)$ returns a solution to Problem $\mathrm{R}\left(I_{0}, I_{1}\right)$.

- $\operatorname{DS}-\operatorname{FIM}(v)$ returns the $D_{s}$ criterion value for the FIM corresponding to $v$.

- Integral-Test $(v)$ returns true only if the current solution $v$ is integral.

- $\operatorname{IndEX}-\operatorname{Branch}(v)$ returns the index defined by (28).

\section{Algorithmic solution for the relaxed problem}

4.1. Problem reformulation. For notational convenience, we replace the variables $v_{i}, i \in I \backslash\left(I_{0} \cup I_{1}\right)$ with $w_{j}, j=1, \ldots, q$, where $q=\left|I \backslash\left(I_{0} \cup I_{1}\right)\right|$, since there exists a bijection $\pi$ from $\{1, \ldots, q\}$ to $I \backslash\left(I_{0} \cup I_{1}\right)$ such that $w_{j}=v_{\pi(j)}, j=1, \ldots, q$. Consequently, we obtain the ultimate formulation:

Problem $\mathbf{R}^{\prime}\left(\boldsymbol{I}_{\mathbf{0}}, \boldsymbol{I}_{\mathbf{1}}\right)$. Find $w \in \mathbb{R}^{q}$ to maximize

$$
\mathcal{Q}(w)=\Psi(G(w))
$$

subject to the constraints

$$
\begin{aligned}
& \sum_{j=1}^{q} w_{j}=r, \\
& 0 \leq w_{j} \leq 1, \quad j=1, \ldots, q,
\end{aligned}
$$




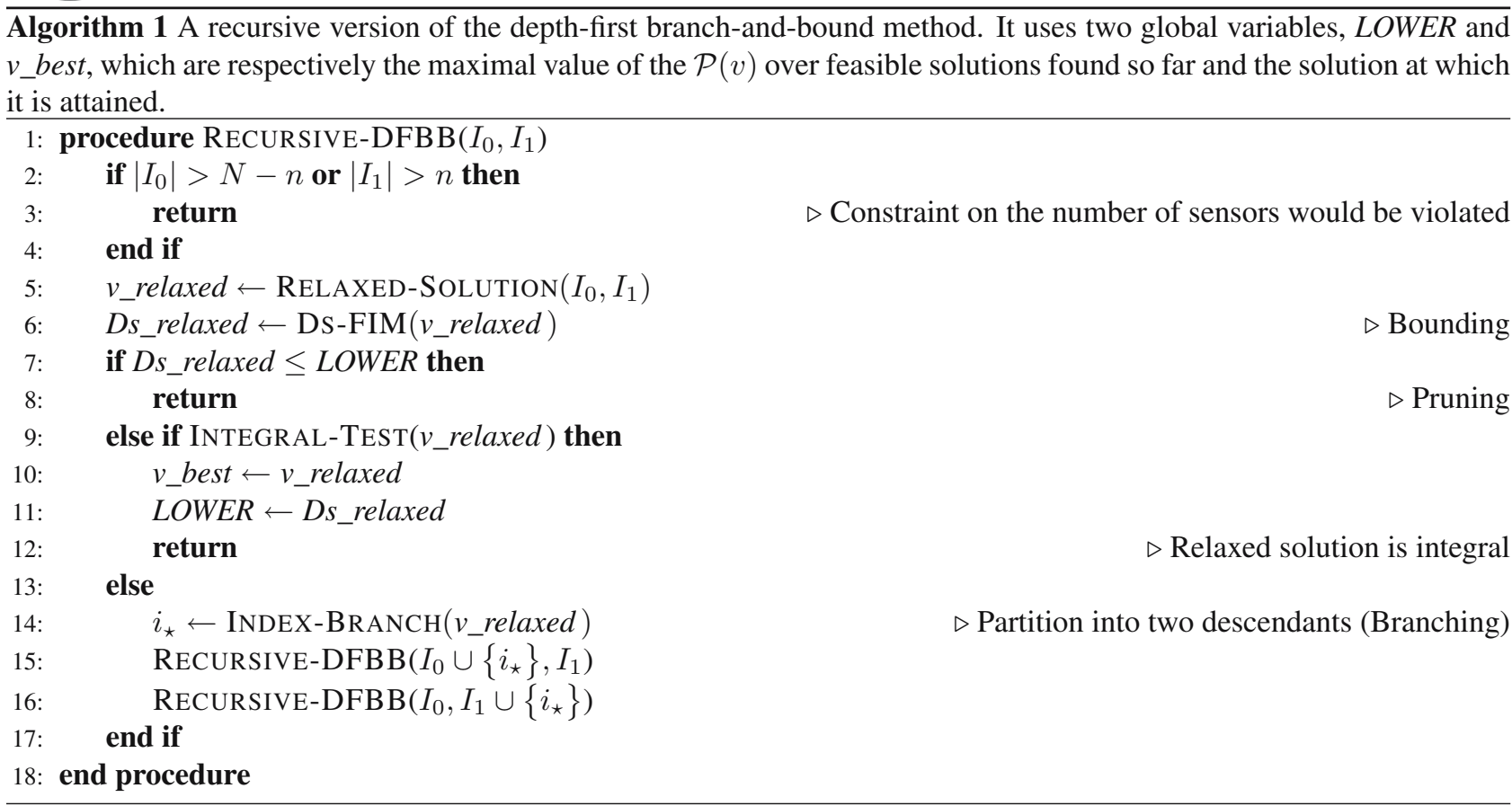

where

$$
\begin{aligned}
& r=n-\left|I_{1}\right|, \quad G(w)=A+\sum_{j=1}^{q} w_{j} S_{j}, \\
& A=\sum_{i \in I_{1}} M_{i}, \quad S_{j}=M_{\pi(j)}
\end{aligned}
$$

for $j=1, \ldots, q$. (Note that $\left|I_{1}\right|$ sensors have already been assigned to locations $x^{i}, i \in I_{1}$, and thus a decision about the placement of $r$ remaining sensors has to be made.)

4.2. Gradient projection scheme. It can be demonstrated (the proof is technical and thus it can be found in Appendix) that the $D_{s}$-optimality criterion is concave over the cone $\mathbb{S}_{++}^{m}$. What is more, its matrix derivative is

$$
\stackrel{\circ}{\Psi}(M)=\frac{\partial \Psi(M)}{\partial M}=\left[\begin{array}{c:c}
D_{\alpha \alpha} & D_{\alpha \beta} \\
\hdashline D_{\alpha \beta}^{\top} & D_{\beta \beta}-M_{\beta \beta}^{-1}
\end{array}\right],
$$

which results from the representation

$$
\begin{aligned}
\Psi[M] & =\log \operatorname{det}(M)-\log \operatorname{det}\left(M_{\beta \beta}\right) \\
& =\log \operatorname{det}(M)-\log \operatorname{det}\left(A^{\top} M A\right),
\end{aligned}
$$

where

$$
A=\left[\begin{array}{c}
0 \\
\cdots \cdots \\
I
\end{array}\right] \in \mathbb{R}^{m \times(m-s)},
$$

and Proposition 10.6.2 of (Bernstein, 2005, p.410).

In the sequel, $W$ will stand for the set of all vectors $w=\left(w_{1}, \ldots, w_{q}\right)$ satisfying (30) and (31). Note that it forms a polygon in $\mathbb{R}^{q}$. The objective function 29 is concave as the composition of the log-determinant with an affine mapping, see (Boyd and Vandenberghe, 2004, p. 79). We wish to maximize it over the polyhedral set $W$.

For that purpose, a number of possibilities exist, cf. (Bertsekas, 1999, Chapter 2), but gradient projection methods prove very efficient. The simplest version is a feasible direction method of the form

$$
w^{k+1}=w^{k}+\lambda^{k}\left(\bar{w}^{k}-w^{k}\right)
$$

where

$$
\bar{w}^{k}=\left[w^{k}+\varrho^{k} \nabla \mathcal{Q}\left(w^{k}\right)\right]^{+} .
$$

Here, $[\cdot]^{+}$denotes projection on the set $W, \lambda^{k} \in(0,1]$ is a stepsize, and $\varrho^{k}$ is a positive scalar. Thus, to obtain the vector $\bar{w}^{k}$, we take a step $\varrho^{k} \nabla \mathcal{Q}\left(w^{k}\right)$ along the gradient, as in steepest ascent. We then project the result $w^{k}+\varrho^{k} \nabla \mathcal{Q}\left(w^{k}\right)$ on $W$, thereby defining the feasible vector $\bar{w}^{k}$. Finally, we take a step along the feasible direction $\bar{w}^{k}-w^{k}$ using the stepsize $\lambda^{k}$.

An $\mathcal{O}(r)$ algorithm of projecting on $W$, which constitutes an intersection of a hyperplane and a box, was set forth by Maculan et al. (2003). Let us also note that

$$
\begin{aligned}
& \nabla \mathcal{Q}(w) \\
& =\left[\operatorname{trace}\left(\stackrel{\circ}{\Psi}(G(w)) S_{1}\right) \ldots \quad \operatorname{trace}\left(\stackrel{\circ}{\Psi}(G(w)) S_{q}\right)\right]^{\top} .
\end{aligned}
$$

\section{Computational results}

As an illustration of the presented approach to the sensor network design, we consider the problem of sensor 
placement for fault detection in the transport process of an air pollutant over a given urban area. Within this domain, which has been normalized to the unit square $\Omega=$ $(0,1)^{2}$, an active source of pollution is present, which influences the pollutant spatial concentration $y=y(x, t)$. The evolution of $y$ over the normalized observation interval $T=(0,1]$ is described by the following advectiondiffusion equation:

$$
\begin{aligned}
\frac{\partial y(x, t)}{\partial t} & +\nabla \cdot(v(x) y(x, t)) \\
& =\nabla \cdot(a(x) \nabla y(x, t))+f(x), \quad x \in \Omega
\end{aligned}
$$

subject to the boundary and initial conditions

$$
\begin{array}{ll}
\frac{\partial y(x, t)}{\partial n}=0, & \text { on } \Gamma \times T, \\
y(x, 0)=y_{0}, & \text { in } \Omega,
\end{array}
$$

where the term $f(x)=50 \exp \left(-50\|x-c\|^{2}\right)$ represents a source of the pollutant located at point $c=(0.3,0.3)$, and $\partial y / \partial n$ stands for the partial derivative of $y$ with respect to the outward normal to the boundary $\Gamma$. The mean spatiotemporal changes in the wind velocity field over the area were approximated by $v=\left(v_{1}, v_{2}\right)$, where

$$
v_{1}=2\left(x_{1}+x_{2}-t\right), \quad v_{2}=x_{2}-x_{1}+t,
$$

which is also illustrated in Fig. 11 The assumed functional form of the spatial-varying diffusion coefficient $a(x)$ is

$$
a(x)=\theta_{1}+\theta_{2} x_{1} x_{2}+\theta_{3} x_{1}^{2}+\theta_{4} x_{2}^{2} .
$$

The subject of interest here is the detection of a significant increase in the intensity of the pollutant emission from the source. As the symptom of this abrupt fault, an excessive deviation of the parameters $\theta_{1}$ and $\theta_{2}$ from their nominal values was assumed. Therefore, these parameters need estimation based on measurement data from monitoring stations.

In our simulation studies, the described branch-andbound technique was applied to determine the locations of stationary sensors. Given $N$ prospective sites in $\Omega \cup \Gamma$, we aim at selecting their subset consisting of the locations at which the measurements made by $n$ available sensors would lead to $D_{s}$-optimum least-squares estimates of the parameters $\theta$.

In order to determine the elements of the sensitivity vector required to calculate the FIM, the direct-differentiation method (Uciński, 2005) was applied assuming the nominal values of the parameters $\theta_{1}^{0}=0.02, \theta_{2}^{0}=0.01$ and $\theta_{3}^{0}=\theta_{4}^{0}=0.005$. We solved the resulting system of PDEs using routines of the MATLAB PDE toolbox for a spatial mesh composed of 682 triangles and 378 nodes. As for the numerical integration required to evaluate information matrices for admissible observation sites, the trapezoidal rule was applied with the time step equal to
0.04 , based on the sensitivity vector interpolated at the nodes representing admissible locations $x^{i}$, cf. Appendix I in (Uciński, 2005) for details.

Complex dynamics of the pollution process are shown in Fig. 11 The pollutant spreads out over the entire domain reflecting the sophisticated combination of diffusion and advection and follows the temporary direction of the wind being the dominant transport factor. In the scenario considered, the observation grid was assumed to be created at locations selected from among those elements of the above-mentioned 378-point triangulation mesh which do not lie on the outer boundary (there were 312 such nodes, which are indicated with dots in Fig. 2).

A Matlab program was written to implement the recursive version of the depth-first branch and bound (DFBB) procedure embodied by Algorithm 1, In order to solve the relaxed problem, which constitutes the principal part of DFBB, the gradient projection scheme was implemented (Sec. 4) with tolerance and maximum number of iterations set to $\epsilon=10^{-8}$ and $\kappa_{\max }=1000$, respectively. Finally, to take full advantage of the efficient gradient projection scheme performed at each node of the BB tree, Algorithm 1 was extended to incorporate a rounding procedure after solving the relaxed problem. Thus, this solution provides not only an upper bound to the currently processed branch, but may also lead to a great improvement in the lower bound (LOWER). Intuitively, a proper way of rounding a relaxed solution is to choose target sites for locating spare sensors so that they correspond to the largest weights. In case this choice is complicated by the presence of sites with identical weights, the target sites are chosen randomly. As a result, it is not necessary to descend to the bottom level of the BB tree in order to update LOWER. This fact is of crucial importance for large-scale problems, where such an action costs many recursive calls of Algorithm 1. Thus the rounding of relaxed solutions significantly makes the algorithm faster as evidenced by numerous simulation experiments.

A well-known fact related to the determination of $D_{s}$-optimum solutions is that the optimal FIM or its diagonal components (10) may be singular. As a result, only some linear combinations or subsets of parameters may be estimable. This is enough for the purpose of verifying the hypothesis (8) related to the detection of the parameter changes considered, but it may lead to numerical difficulties in the implementation of the gradient projection algorithm presented in Section 4.2 Therefore, for numerical construction of sensor network configurations, some simple regularization techniques for the FIM should be applied (Atkinson et al., 2007).

$D_{s}$-optimal sensor configurations for different numbers of allocated sensors are shown in Fig. 2. It is clear that the complexity of the system dynamics makes the proper prediction of the observation locations rather difficult and nonintuitive. The sensors tend to form a pat- 


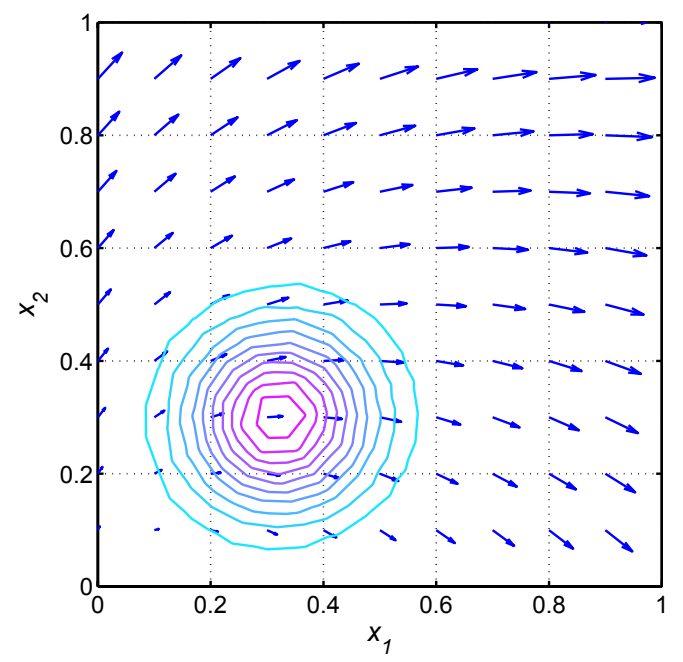

(a) $t=0$

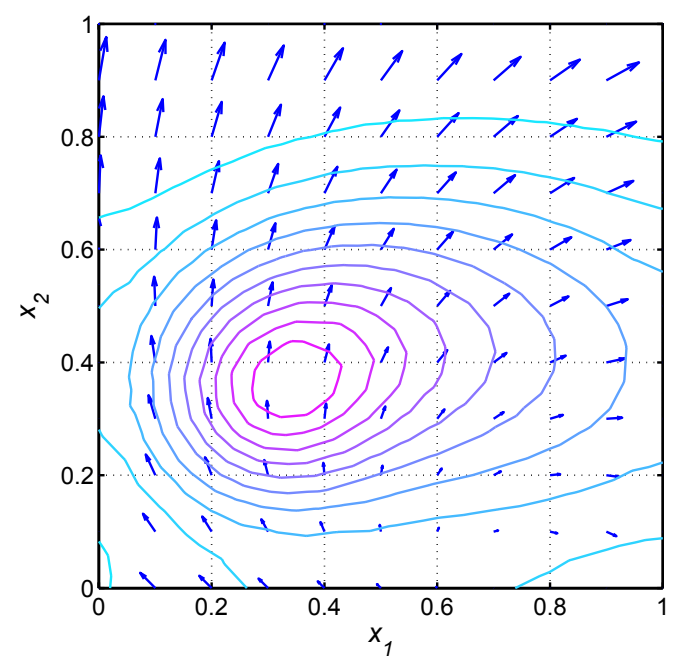

(c) $t=0.7$

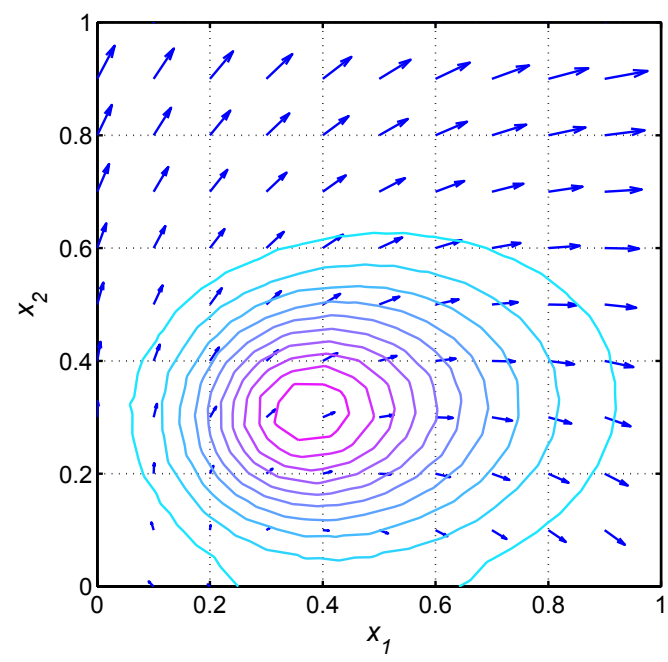

(b) $t=0.3$

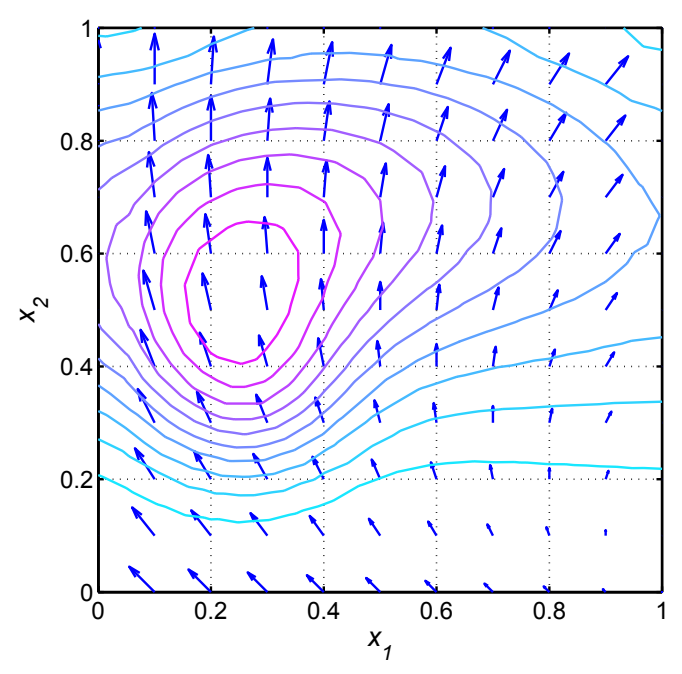

(d) $t=1$

Fig. 1. Temporal changes in the wind velocity field and pollutant concentration.

tern reflecting the areas of greatest changes in the pollutant concentration but the observations are averaged over time and it is not trivial to follow the dynamics of the observation strategy. Surprisingly, the measurements in the closest vicinity of the pollution source turned out to be not very attractive for the fault detection considered. The results concerning the algorithm performance are summarized in Tab. 1. The number of recursive calls of the DFBB procedure equals to one, which means that the optimal solution is obtained just by rounding the fully relaxed problem. The examination of the data from Tab. 1 leads to interesting conclusions. Unexpectedly, with an increased number of sensors (and the size of the corresponding search space), the pruning process becomes more efficient. This effect can be explained by observing that a higher density of sensors leads to a better estimate of the lower bound to the optimal value of the design criterion, which results in an increased efficiency of pruning and whereupon the search is speeded up.

\section{Conclusions}

We consider the problem of monitoring network design which provides proper diagnostic information about the state of the distributed parameter system considered. One of the most important issues related to this task is the choice of a suitable criterion to quantify the quality of the detection of an abnormal system state indicating potential faults. We state the problem in the form of the maximization of the power for a parametric hypothesis test which verifies the nominal values for the system pa- 


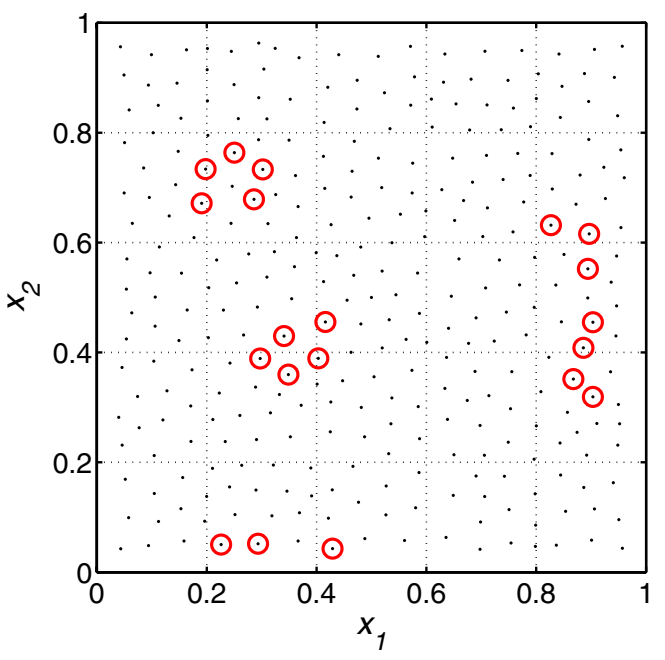

(a) $n=20$

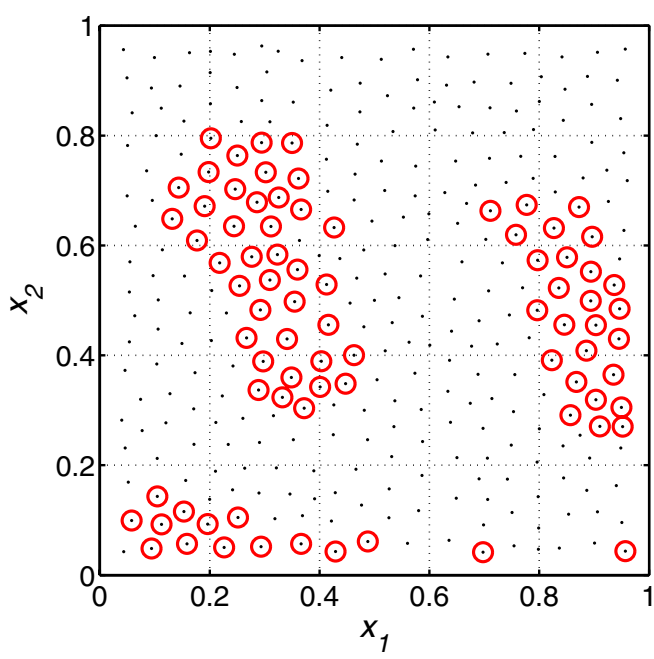

(c) $n=80$

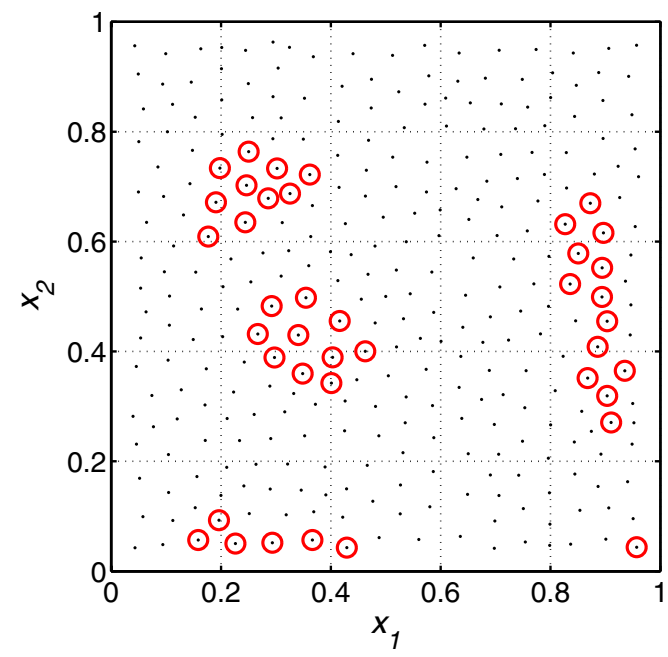

(b) $n=40$



(d) $n=150$

Fig. 2. $D_{s}$-optimal allocation of different numbers of sensors.

rameters. Then, as an appropriate performance index, the $D_{s}$-optimality criterion defined on the Fisher information matrix is proposed. Although this criterion is well known in optimum design theory, there exist only few attempts to exploit it in the context of fault detection for DPSs, cf. (Patan, 2004; Patan and Patan, 2005; Patan et al., 2005; Uciński, 2003). This work contributes to this issue providing a proper mathematical justification of some valuable properties of $D_{s}$-optimality such as its concavity and differentiability.

Another crucial difficulty here is the large scale of the resulting global optimization problem, since the monitoring networks encountered in process industry or environmental engineering may often consist of several hundreds of stations. Obviously, this makes the exhaustive search on a candidate-by-candidate basis practically in- tractable and creates a need for techniques which would implement a guided search and have acceptable performance. With our sensor network design problem, we started from the most common formulation, in which the measurement system has a finite number of candidate sensor positions and the aim is to select the best subset of points (of a desired cardinality) in the sense of maximizing the $D_{s}$-optimality criterion. This fits into the framework of nonlinear $0-1$ integer programming. The solution of this combinatorial design problem using the branchand-bound method constitutes quite a natural option, but the main problem when trying to implement it has been the lack of a low-cost procedure to obtain upper bounds to the optimal values of the $D_{s}$-optimality criterion. Our main contribution consists in adapting a specialized gradient projection procedure for criterion maximization to 
Table 1. Comparison of algorithm performance for different settings.

\begin{tabular}{|c|ccc|}
\hline $\begin{array}{c}\text { No. of } \\
\text { sensors }\end{array}$ & $\begin{array}{c}\text { No. of recur- } \\
\text { sive calls }\end{array}$ & $\begin{array}{c}\text { Time } \\
{[\mathrm{s}]}\end{array}$ & $\begin{array}{c}\text { Criterion } \\
\text { value }\end{array}$ \\
\hline \hline 10 & 17 & 4.697 & $1.19422 \mathrm{e}+006$ \\
20 & 41 & 9.043 & $4.28668 \mathrm{e}+006$ \\
40 & 5 & 3.856 & $1.59385 \mathrm{e}+007$ \\
60 & 1 & 1.092 & $3.33651 \mathrm{e}+007$ \\
80 & 1 & 0.511 & $5.8198 \mathrm{e}+007$ \\
100 & 3 & 1.698 & $8.24685 \mathrm{e}+007$ \\
120 & 1 & 0.261 & $1.14538 \mathrm{e}+008$ \\
150 & 7 & 3.395 & $1.59447 \mathrm{e}+008$ \\
\hline
\end{tabular}

produce such bounds. Consequently, the proposed method can be implemented with ease and our experience provides evidence that, with this tool, even large-scale design problems can be solved using an off-the-shelf PC.

Naturally, there still remain some open problems which need close attention. The following points can be raised as the main directions of further research:

- Extension of the discussed class of DPSs. The great advantage of the delineated approach is that it is independent of a specific form of PDEs used as a mathematical model of the DPS considered. In such a manner the presented approach can be rather easily adopted for the class of multi-output DPSs or/and systems with delays, since only the formula for calculating the elements of the FIM will be suitably changed.

- The structure of the proposed BB algorithm is well suited for parallel implementation dedicated to clusters of PCs. Such a parallel version of the approach would be very attractive for solving large-scale problems.

- The proposed simple branching rule for the binary $\mathrm{BB}$ tree can be refined by incorporating a mechanism driving the search proces towards the most promising branches in terms of the objective function or heuristics exploiting specific properties of the DPS considered.

\section{Appendix}

Proof of the concavity of the $D_{s}$-optimality criterion Recall that the Euclidean matrix scalar product

$$
\langle G, H\rangle=\operatorname{trace}\left(G^{\top} H\right)
$$

turns $\mathbb{R}^{m \times m}$ into a Euclidean space of dimension $m^{2}$. We denote by $\mathbb{S}^{m}$ its subspace of symmetric matrices.
It is well known that this subspace contains the convex, pointed and closed cone of nonnegative definite matrices, $\mathbb{S}_{+}^{m}$, which has the set of positive definite matrices, $\mathbb{S}_{++}^{m}$, as its interior relative to the space $\mathbb{S}^{m}$, cf. (Pukelsheim, 1993, p. 10).

The Löwner partial ordering $\succeq$ of $\mathbb{S}^{m}$ is defined as follows:

$$
G \succeq H \Leftrightarrow G-H \succeq 0 \Leftrightarrow G-H \in \mathbb{S}_{+}^{m} .
$$

Recall that

$$
G \in \mathbb{S}_{+}^{m} \Leftrightarrow \operatorname{trace}(G H) \succeq 0, \quad \forall H \in \mathbb{S}_{+}^{m} .
$$

Lemma 1. If $G \succeq H \succeq 0$, then for all $V \in \mathbb{S}^{m}$ we have

$$
\operatorname{trace}(G V G V) \geq \operatorname{trace}(H V H V) \text {. }
$$

Proof. From (3) it follows that

$$
\operatorname{trace}(G V G V) \geq \operatorname{trace}(G V H V) \geq \operatorname{trace}(H V H V) \text {. }
$$

The two inequalities result from $V G V \succeq V H V$ and $G \succeq$ $H$, respectively.

Lemma 2. For the partition (10), if $M \in \mathbb{S}_{++}^{m}$, then

$$
M^{-1} \succeq A\left(A^{\top} M A\right)^{-1} A^{\top},
$$

where $A$ is defined in (36).

Proof. First observe that, given $M \in \mathbb{S}_{++}^{m}$, we have $M_{\beta \beta}=A^{\top} M A \in \mathbb{S}_{++}^{m}$ whenever $s<m$.

From Proposition 2.8.7 of (Bernstein, 2005, p.44) we deduce that

$$
\begin{aligned}
& M^{-1}-A\left(A^{\top} M A\right)^{-1} A^{\top} \\
& =\left[\begin{array}{c:c}
D_{\alpha \alpha} & -D_{\alpha \alpha} M_{\alpha \beta} M_{\beta \beta}^{-1} \\
\hdashline-M_{\beta \beta}^{-1} M_{\alpha \beta}^{\top} D_{\alpha \alpha} & M_{\beta \beta}^{-1} M_{\alpha \beta}^{\top} D_{\alpha \alpha} M_{\alpha \beta} M_{\beta \beta}^{-1}
\end{array}\right] .
\end{aligned}
$$

Then for any $y \in \mathbb{R}^{m}$ partitioned as

$$
y=\left[\begin{array}{c}
y_{\alpha} \\
\cdots \\
y_{\beta}
\end{array}\right]
$$

we obtain

$$
\begin{aligned}
& y^{\top}\left(M^{-1}-A\left(A^{\top} M A\right)^{-1} A^{\top}\right) y \\
& \quad=\left(y_{\alpha}-M_{\alpha \beta} M_{\beta \beta}^{-1} y_{\beta}\right)^{\top} D_{\alpha \alpha}\left(y_{\alpha}-M_{\alpha \beta} M_{\beta \beta}^{-1} y_{\beta}\right) \\
& \quad \geq 0,
\end{aligned}
$$

which yields the desired result. 
We claim that the criterion (9) is concave over $\mathbb{S}_{++}^{m}$. Indeed, denoting by $\Psi_{s}^{\prime}(M)$ the first Fréchet derivative of $\Psi_{s}$ at $M \in \mathbb{S}_{++}^{m}$, from (35) and Proposition 10.6.2(vi) in (Bernstein, 2005, p.410) we obtain

$$
\begin{aligned}
\Psi_{s}^{\prime} & (M) V \\
& =\operatorname{trace}\left\{\frac{\partial}{\partial M}\left[\Psi_{s}(M)\right] V\right\} \\
& =\operatorname{trace}\left\{\left[M^{-1}-A\left(A^{\top} M A\right)^{-1} A^{\top}\right] V\right\}
\end{aligned}
$$

for any $V \in \mathbb{S}^{m}$.

As for the second Fréchet derivative of $\Psi_{s}$ at $M \in$ $\mathbb{S}_{++}^{m}$, denoted by $\Psi_{s}^{\prime \prime}(M)$, its value $\Psi_{s}^{\prime \prime}(M)(U, V)$ at given $U, V \in \mathbb{S}^{m}$ equals the derivative of the mapping $M \mapsto$ $\Psi_{s}^{\prime}(M) V$ applied to $U$, i.e.,

$$
\begin{aligned}
\Psi_{s}^{\prime \prime}(M) & (U, V) \\
= & \operatorname{trace}\left\{\frac{\partial}{\partial M}\left[\Psi_{s}^{\prime}(M) V\right] U\right\} \\
= & -\operatorname{trace}\left\{\left[M^{-1} V M^{-1}\right.\right. \\
& \left.\left.-A\left(A^{\top} M A\right)^{-1} A^{\top} V A\left(A^{\top} M A\right)^{-1} A^{\top}\right] U\right\},
\end{aligned}
$$

the last equality resulting from Proposition 10.6.4(iii) in (Bernstein, 2005, p.411).

From Lemmas 1 and 2 we then get

$$
\begin{aligned}
\Psi_{s}^{\prime \prime}(M) & (V, V) \\
= & -\operatorname{trace}\left\{M^{-1} V M^{-1} V\right\} \\
& +\operatorname{trace}\left\{A\left(A^{\top} M A\right)^{-1} A^{\top} V A\left(A^{\top} M A\right)^{-1} A^{\top} V\right\} \\
\leq & 0, \quad \forall V \in \mathbb{S}^{m},
\end{aligned}
$$

which establishes the concavity of $\Psi_{s}$.

\section{Acknowledgment}

The work was supported by the Polish Ministry of Science and Higher Education under Grant No. NN519 297133.

\section{References}

Amouroux M. and Babary J. P. (1988). Sensor and control location problems, in M. G. Singh (Ed), Systems \& Control Encyclopedia. Theory, Technology, Applications, Vol. 6, Pergamon Press, Oxford, pp. 4238-4245.

Atkinson A. C., Donev A. N. and Tobias R. (2007). Optimum Experimental Design, with SAS, Oxford University Press, Oxford.

Bernstein D. S. (2005). Matrix Mathematics. Theory, Facts, and Formulas with Application to Linear Systems Theory, Princeton University Press, Princeton, NJ.

Bertsekas D. P. (1999). Nonlinear Programming, 2nd Edn, Athena Scientific, Belmont, MA.
Boer E. P. J., Hendrix E. M. T. and Rasch D. A. M. K. (2001). Optimization of monitoring networks for estimation of the semivariance function, in A. C. Atkinson, P. Hackl and W. Müller (Eds), mODa 6, Proceedings of the 6th International Workshop on Model-Oriented Data Analysis, Puchberg/Schneeberg, Austria, Physica-Verlag, Heidelberg, pp. 21-28.

Boyd S. and Vandenberghe L. (2004). Convex Optimization, Cambridge University Press, Cambridge.

Cassandras C. G. and Li W. (2005). Sensor networks and cooperative control, European Journal of Control 11(4-5): 436463.

Chiang L. H., Russell E. L. and Braatz R. D. (2001). Fault Detection and Diagnosis in Industrial Systems, Springer-Verlag, London.

Fedorov V. V. and Hackl P. (1997). Model-Oriented Design of Experiments, Lecture Notes in Statistics, Springer-Verlag, New York, NY.

Floudas C. A. (2001). Mixed integer nonlinear programming, MINLP, in C. A. Floudas and P. M. Pardalos (Eds), Encyclopedia of Optimization, Kluwer Academic Publishers, Dordrecht, Vol. 3, pp. 401-414.

Gerdts M. (2005). Solving mixed-integer optimal control problems by branch\&bound: A case study from automobile test-driving with gear shift, Journal of Optimization Theory and Applications 26: 1-18.

Isermann R. (1997). Supervision, Fault Detection and Diagnosis of Technical Systems, Control Engineering Practice 5(5): 639-652.

Korbicz J., Kościelny J., Kowalczuk Z. and Cholewa W. (2004). Fault Diagnosis. Models, Artificial Intelligence, Applications, Springer-Verlag, Berlin.

Kubrusly C. S. and Malebranche H. (1985). Sensors and controllers location in distributed systems-A survey, Automatica 21(2): 117-128.

Maculan N., Santiago C. P., Macambira E. M. and Jardim M. H. C. (2003). An $O(n)$ algorithm for projecting a vector on the intersection of a hyperplane and a box in $\mathbb{R}^{n}$, Journal of Optimization Theory and Applications 117(3): 553-574.

Omatu S. and Seinfeld J. H. (1989). Distributed Parameter Systems: Theory and Applications, Oxford University Press, New York, NY.

Patan M. (2004). Optimal Observation Strategies for Parameter Estimation of Distributed Systems, Zielona Góra University Press. Accessible at http: / /www . zbc. zgora.pl.

Patan M. and Patan K. (2005). Optimal observation strategies for model-based fault detection in distributed systems, International Journal of Control 78(18): 1497-1510.

Patan M., Uciński D. and Baranowski P. (2005). Optimal observation strategies for fault detection in distributedparameter systems, Pomiary, Automatyka, Kontrola (9): 71-73.

Patton R. J., Frank P. M. and Clark R. (2000). Issues of Fault Diagnosis for Dynamic Systems, Springer-Verlag, Berlin. 
Patton R. J. and Korbicz J. (Eds.) (1999). Advances in Computational Intelligence, International Journal of Applied Mathematics and Computer Science 9 (3).

Pázman A. (1986). Foundations of Optimum Experimental Design, D. Reidel Publishing Company, Dordrecht.

Pukelsheim F. (1993). Optimal Design of Experiments, John Wiley \& Sons, New York, NY.

Quereshi Z. H., Ng T. S. and Goodwin G. C. (1980). Optimum experimental design for identification of distributed parameter systems, International Journal of Control 31(1): $21-29$.

Rafajłowicz E. (1981). Design of experiments for eigenvalue identification in distributed-parameter systems, International Journal of Control 34(6): 1079-1094.

Rafajłowicz E. (1983). Optimal experiment design for identification of linear distributed-parameter systems: Frequency domain approach, IEEE Transactions on Automatic Control 28(7): 806-808.

Reinefeld A. (2001). Heuristic search, in C. A. Floudas and P. M. Pardalos (Eds), Encyclopedia of Optimization, Kluwer Academic Publishers, Dordrecht, Vol. 2, pp. 409-411.

Russell S. J. and Norvig P. (2003). Artificial Intelligence: A Modern Approach, 2nd Edn, Pearson Education International, Upper Saddle River, NJ.

Sun N.-Z. (1994). Inverse Problems in Groundwater Modeling, Kluwer Academic Publishers, Dordrecht.

Uciński D. (1992). Optimal sensor location for parameter identification of distributed systems, International Journal of Applied Mathematics and Computer Science 2(1): 119-134.
Uciński D. (1999). Measurement Optimization for Parameter Estimation in Distributed Systems, Technical University Press, Zielona Góra.

Available at http://www.zbc.zgora.pl.

Uciński D. (2000). Optimal selection of measurement locations for parameter estimation in distributed processes, International Journal of Applied Mathematics and Computer Science 10(2): 357-379.

Uciński D. (2003). On optimum experimental design for technical diagnostics of processes, in Z. Kowalczuk (Ed), Proceedings of 6-th National Conference on Diagnostics of Industrial Processes, Władysławowo, Poland, Technical University Press, Gdańsk, pp. 207-212, (in Polish).

Uciński D. (2005). Optimal Measurement Methods for Distributed-Parameter System Identification, CRC Press, Boca Raton, FL.

Uciński D. and Patan M. (2007). D-optimal design of a monitoring network for parameter estimation of distributed systems, Journal of Global Optimization 39(2): 291-322.

van de Wal M. and de Jager B. (2001). A review of methods for input/output selection, Automatica 37(4): 487-510.

Walter É. and Pronzato L. (1997). Identification of Parametric Models from Experimental Data, Communications and Control Engineering, Springer-Verlag, Berlin.

Received: 22 January 2008 Revised: 23 May 2008 